\title{
Atypical Cardiac Tamponade Manifesting as Left Ventricular Diastolic Collapse: A Case Report
}

\author{
Shweta Paulraj ${ }^{1}$, Vijay Raj ${ }^{2}$, Prashanth Ashok Kumar ${ }^{1}$, Robert Voelker $^{2}$, Harold Smulyan ${ }^{2}$ \\ 1. Internal Medicine, State University of New York Upstate Medical University, Syracuse, USA 2. Cardiology, State \\ University of New York Upstate Medical University, Syracuse, USA
}

Corresponding author: Shweta Paulraj, paulrajs@upstate.edu

\begin{abstract}
Cardiac tamponade is a medical emergency, the diagnosis of which is predominantly clinical with supportive echocardiographic findings. Echocardiographic findings highly suggestive of cardiac tamponade include chamber collapse, inferior vena cava (IVC) plethora, and respiratory volume/flow variations. The right-sided cardiac chambers are a low-pressure system and are the first to show signs of collapse with high specificity for tamponade. We report the case of a 35-year-old woman who demonstrated left ventricular (LV) diastolic collapse on echocardiogram following a tricuspid valve replacement. Although left-sided chamber collapse with tamponade has been reported with localized pericardial effusions postoperatively, our patient had a large circumferential pericardial effusion. Selective chamber compression can be a presenting sign of postoperative tamponade after cardiac surgery. Our case highlights the importance of recognizing atypical forms of cardiac tamponade to help in early identification and emergent management in such patients.
\end{abstract}

Categories: Cardiac/Thoracic/Vascular Surgery, Cardiology, Internal Medicine

Keywords: pericardial effusion, echocardiogram, valve replacement, tamponade, cardiac surgery, diastolic collapse, chamber collapse, paradoxical septal motion, hemopericardium

\section{Introduction}

Cardiac tamponade is a medical emergency that requires timely recognition and treatment [1]. It is predominantly a clinical diagnosis with Beck's triad, comprising hypotension, distended neck veins, and muffled heart sounds, present only in a minority of patients [2]. Echocardiography aids in the diagnosis of cardiac tamponade by confirming the presence of fluid and using chamber collapse to assess its hemodynamic impact [3]. Early diastolic collapse of the right-sided chambers is a frequent and specific finding for cardiac tamponade, but the collapse of the left ventricle (LV) is rare [4]. We report the case of a patient with cardiac tamponade manifesting as LV diastolic chamber compression on echocardiography.

Received 04/20/2020 Review began 04/25/2020 Review ended 05/01/2020 Published 05/10/2020

(c) Copyright 2020 Paulraj et al. This is an open access article distributed under the terms of the Creative Commons Attribution License CC-BY 4.0., which permits unrestricted use, distribution, and reproduction in any medium, provided the original author and source are credited.

\section{Case Presentation}

A 35-year-old African-American woman with sickle cell disease presented to the hospital with severe, progressive dyspnea and a sickle cell crisis. She had a history of severe tricuspid regurgitation and had been previously treated for protein $\mathrm{C}$ deficiency, recurrent deep vein thrombosis, and pulmonary emboli with an inferior vena cava (IVC) filter and chronic warfarin therapy. On examination, she had $2+$ pitting pedal edema, jugular venous distension, a grade $2 / 6$ systolic murmur over the tricuspid area, tender hepatomegaly, and diffuse abdominal distension. Echocardiogram revealed severe tricuspid regurgitation, a markedly dilated right atrium, dilated tricuspid annulus, and a mildly dilated but normally functioning right ventricle (RV). Doppler findings suggested moderate pulmonary hypertension with a pulmonary artery systolic pressure of $55 \mathrm{mmHg}$. These findings led the doctors to conduct a bioprosthetic tricuspid valve replacement surgery.

Postoperatively, she was hemodynamically stable, had crackles on lung auscultation and hypoxic respiratory failure that required 2-3 L/minute of oxygen via a nasal cannula, intermittent bilevel positive airway pressure ventilation, and diuresis. Twenty days after surgery, purulent secretion was noted on the sternotomy site. A CT of the thorax showed a large pericardial effusion, possibly a hemopericardium, and bilateral pleural effusions. Warfarin was discontinued. The echocardiogram showed a large circumferential pericardial effusion, predominantly posterior in a location with fibrinous strands (Figure 1, Video 1). 


\section{Cureus}

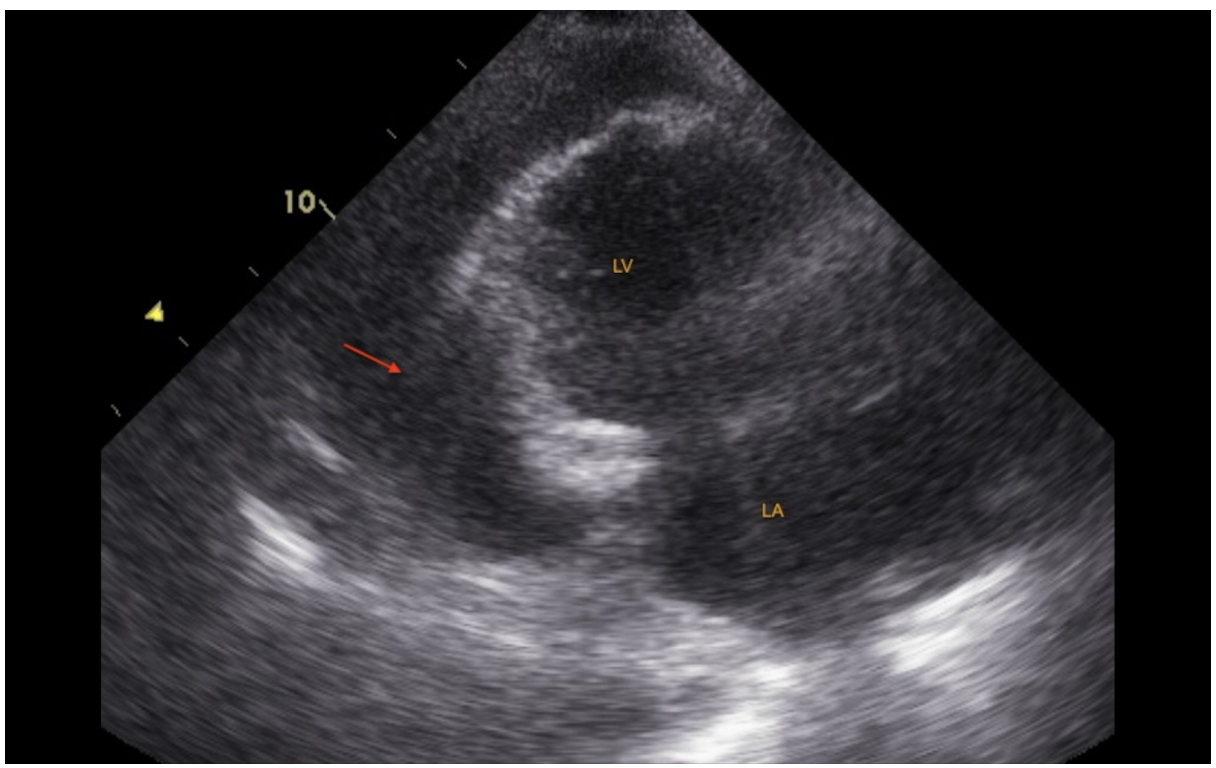

FIGURE 1: Transthoracic echocardiogram: apical two-chamber view

The image shows large circumferential pericardial effusion, predominantly posterior in a location with fibrinous strands (arrow)

LA: left atrium; LV: left ventricle

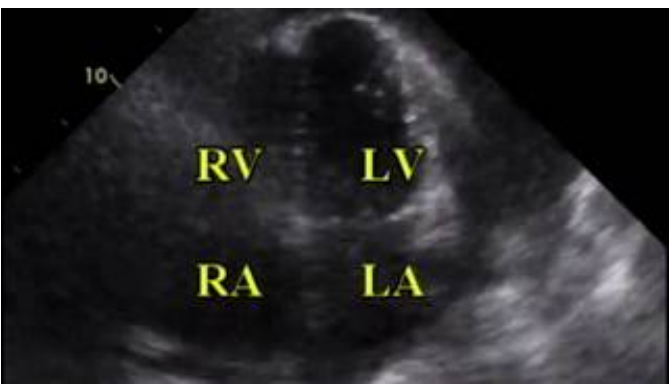

VIDEO 1: Transthoracic echocardiogram: apical four-chamber view showing LV collapse

The video shows large circumferential pericardial effusion with diastolic collapse of the LV

LA: left atrium; LV: left ventricle; RA: right atrium; RV: right ventricle

View video here: https://www.youtube.com/watch?v=kHpF9Sut5Dw

There was paradoxical interventricular septal motion with LV septal bounce, excessive mitral inflow respiratory driven variation of $\sim 40 \%$ (Figure 2), and LV apical diastolic compression (Figure 3, Video 1). The prosthetic tricuspid valve functioned normally. 


\section{Cureus}

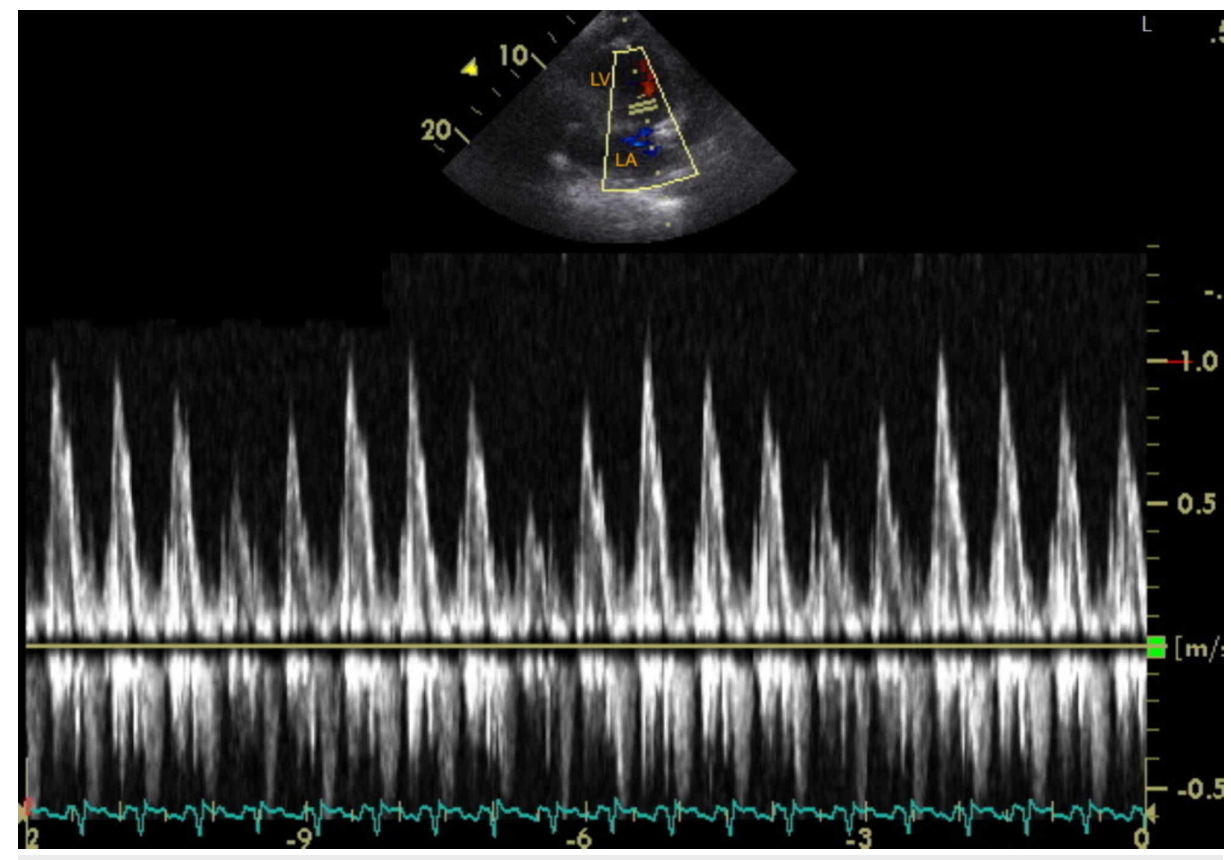

FIGURE 2: Transthoracic echocardiogram with pulse wave doppler

The image shows mitral inflow velocity on pulse wave doppler showing about $40 \%$ respiratory-driven variation

LA: left atrium; LV: left ventricle

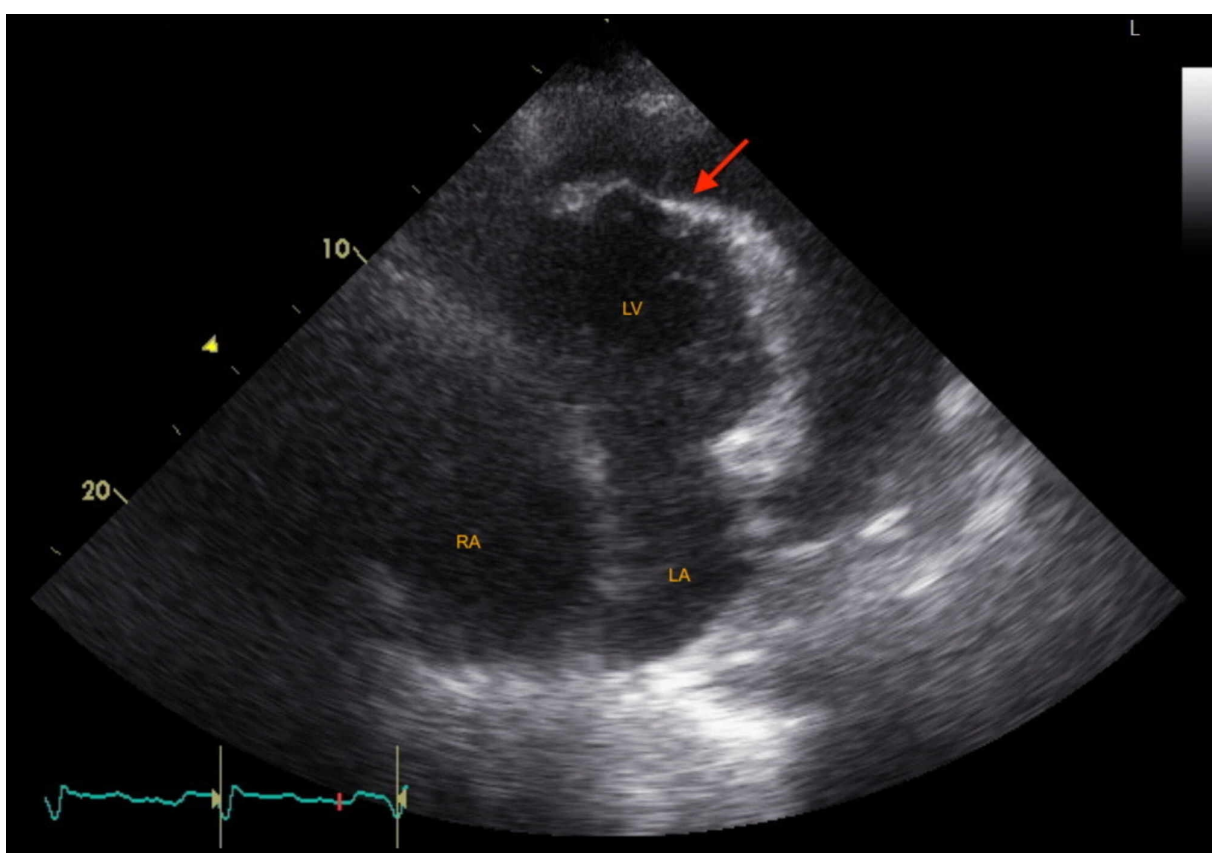

FIGURE 3: Transthoracic echocardiogram with LV collapse

Apical-four chamber view showing large pericardial effusion with diastolic LV apical collapse (arrow)

LA: left atrium; LV: left ventricle; RA: right atrium

She underwent a subxiphoid pericardial window and placement of a drainage catheter, which led to the removal of 500 cubic centimeters of blood followed by a gradual improvement in her symptoms. A follow-up echocardiogram two months after discharge showed no pericardial effusion (Figure 4). 


\section{Cureus}

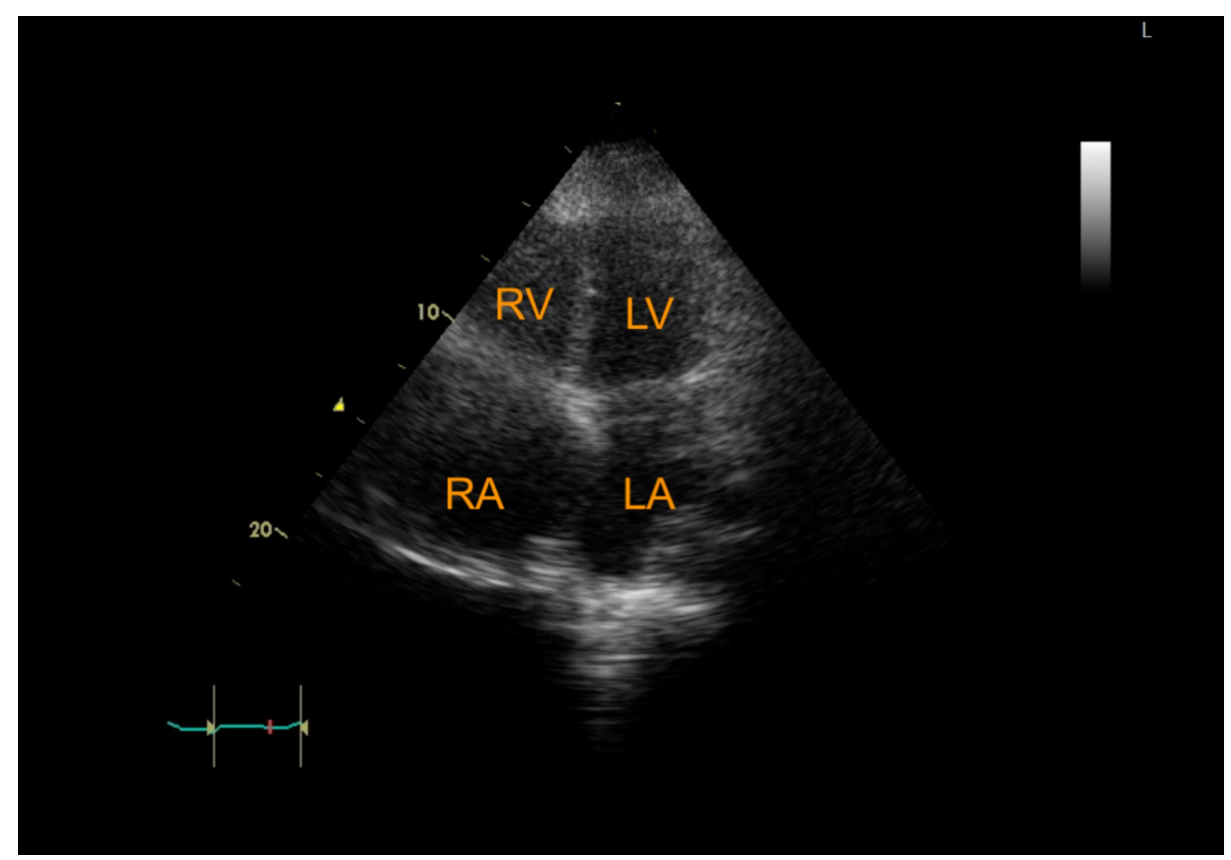

FIGURE 4: Postoperative echocardiogram with no pericardial effusion

Apical four-chamber view showing RA, RV, LA, and LV

LA: left atrium; LV: left ventricle; RA: right atrium; RV: right ventricle

\section{Discussion}

Pericardial effusions can be secondary to infections, inflammatory diseases, infarction, malignancy, valve replacements, and thoracotomy [5]. Effusions with cardiac tamponade are more common following valve surgery than after coronary artery bypass graft surgery, with a reported incidence of $0.6-4.3 \%$ [6]. Various causative mechanisms have been postulated, the chief one being anticoagulation. Postoperative cardiac tamponade is reported to be more common in females, and any amount of pericardial effusion on the first postoperative transthoracic echocardiogram has been found to be predictive of cardiac tamponade. Despite prolonged hospital stays and high readmission rates in patients with postoperative cardiac tamponade, its course is usually benign if intervened in a timely manner [7].

Significant echocardiographic findings in tamponade include chamber collapse, commonly of the rightsided chambers, IVC plethora, and excessive respiratory variation in cardiac inflow volume. The intrapericardial pressure exceeds the chamber pressure in patients with tamponade, and the low-pressure right-sided chambers usually show the first signs of collapse [8]. The LV is thicker, less compliant, and encloses a high-pressure system that tends to resist collapse [3]. Interventricular septal bounce on the echocardiogram is highly suggestive of cardiac tamponade with increased ventricular interdependence [9].

LV diastolic collapse has been described as a sign of cardiac tamponade following cardiac surgery [10]. These effusions can be located posteriorly in $49 \%$ of the patients but also may be compartmentalized, causing a diastolic collapse of the LV rather than the RV [6]. Loculated effusions due to adhesions can cause the typical hemodynamic abnormalities in the compressed chambers only and therefore posterior pericardial effusions can lead to isolated LV cardiac tamponade [11]. Another scenario that could explain the left side chamber collapse is pulmonary artery hypertension where increased right heart pressures may exceed pericardial pressures and prevent right-sided collapse. Left-sided filling pressures may then be lower than pericardial pressures in such patients, producing collapse of the left atrium [3]. Our patient had evidence of moderate pulmonary hypertension in her initial echocardiogram.

Echocardiographic detection of chamber collapse, increased respiratory-driven valvular flow variation, and reduced hepatic venous flow all suggest imminent cardiovascular collapse and require urgent treatment. Echocardiographic guided pericardiocentesis and the removal of even a small amount of pericardial fluid can result in a substantial drop in the intrapericardial pressure and improvement in symptoms [8]. Catheter drainage of the pericardial fluid and surgical drainage via a pericardial window are other options in patients with hemopericardium (as in our patient), purulent pericarditis, or neoplastic effusions with a high risk for recurrence $[12,13]$. 


\section{Conclusions}

Atypical forms of cardiac tamponade with varied clinical presentations may be seen in patients after cardiac surgery, often with selective chamber compression. An awareness of these uncommon presentations of cardiac tamponade is essential for prompt and appropriate emergency care of these patients.

\section{Additional Information}

\section{Disclosures}

Human subjects: Consent was obtained by all participants in this study. Conflicts of interest: In compliance with the ICMJE uniform disclosure form, all authors declare the following: Payment/services info: All authors have declared that no financial support was received from any organization for the submitted work. Financial relationships: All authors have declared that they have no financial relationships at present or within the previous three years with any organizations that might have an interest in the submitted work. Other relationships: All authors have declared that there are no other relationships or activities that could appear to have influenced the submitted work.

\section{References}

1. Ristić AD, Imazio M, Adler Y, et al.: Triage strategy for urgent management of cardiac tamponade: a position statement of the European Society of Cardiology Working Group on Myocardial and Pericardial Diseases. Eur Heart J. 2014, 35:2279-2284. 10.1093/eurheartj/ehu217

2. Shabetai R: Pericardial and cardiac pressure. Circulation. 1988, 77:1-5. 10.1161/01.cir.77.1.1

3. Adler Y, Charron P, Imazio M, et al.: 2015 ESC Guidelines for the diagnosis and management of pericardial diseases: The Task Force for the Diagnosis and Management of Pericardial Diseases of the European Society of Cardiology (ESC) Endorsed by: The European Association for Cardio-Thoracic Surgery (EACTS). Eur Heart J. 2015, 36:2921-2964. 10.1093/eurheartj/ehv318

4. Singh S, Wann LS, Schuchard GH, Klopfenstein HS, Leimgruber PP, Keelan MH Jr, Brooks HL: Right ventricular and right atrial collapse in patients with cardiac tamponade--a combined echocardiographic and hemodynamic study. Circulation. 1984, 70:966-971. 10.1161/01.cir.70.6.966

5. Kearns MJ, Walley KR: Tamponade: hemodynamic and echocardiographic diagnosis. Chest. 2018, 153:12661275. 10.1016/j.chest.2017.11.003

6. Kuvin JT, Harati NA, Pandian NG, Bojar RM, Khabbaz KR: Postoperative cardiac tamponade in the modern surgical era. Ann Thorac Surg. 2002, 74:1148-1153. 10.1016/S0003-4975(02)03837-7

7. You SC, Shim CY, Hong GR, et al.: Incidence, predictors, and clinical outcomes of postoperative cardiac tamponade in patients undergoing heart valve surgery. PLoS One. 2016, 11:e0165754. Accessed: May 8, 2020: https://www.ncbi.nlm.nih.gov/pmc/articles/PMC5113894/. 10.1371/journal.pone.0165754

8. Appleton C, Gillam L, Koulogainnis K: Cardiac tamponade. Cardiol Clin. 2017, 35:525-537. 10.1016/j.ccl.2017.07.006

9. Walker CM, Chung JH, Reddy GP: "Septal bounce". J Thorac Imaging. 2012, 27:W1. 10.1097/RTI.0b013e31823fdfbd

10. Chuttani K, Pandian NG, Mohanty PK, et al.: Left ventricular diastolic collapse. An echocardiographic sign of regional cardiac tamponade. Circulation. 1991, 83:1999-2006. 10.1161/01.CIR.83.6.1999

11. Spodick DH: Acute cardiac tamponade. N Engl J Med. 2003, 349:684-690. 10.1056/NEJMra022643

12. Levis JT, Delgado MC: Hemopericardium and cardiac tamponade in a patient with an elevated international normalized ratio. West J Emerg Med. 2009, 10:115-119.

13. Sagristà-Sauleda J, Mercé AS, Soler-Soler J: Diagnosis and management of pericardial effusion. World J Cardiol. 2011, 3:135-143. Accessed: May 8, 2020: https://www.ncbi.nlm.nih.gov/pmc/articles/PMC3110902/. 10.4330/wjc.v3.i5.135 